\title{
¿Podemos ser "racionales" al comprar una casa? Una visión desde la Economía Conductual
}

\author{
Guillermo GARCÍA-BADELL \\ Universidad Politécnica de Madrid-Centro Superior de Diseño de Moda de Madrid, \\ gacademica.csdmm@upm.es \\ Manuel BLANCO \\ Universidad Politécnica de Madrid-Escuela Técnica Superior de Arquitectura de Madrid \\ director.academico.csdmm@upm.es
}

Recibido: $10-10-2016$

Aceptado: 21-10-2016

\section{RESUMEN}

Las teorías económicas tradicionales consideran que nuestras decisiones de compra son racionales, más si cabe cuando éstas son trascendentales para nuestro presupuesto y para nuestro tipo de vida. Comprar una casa requeriría entonces el mayor de nuestros esfuerzos pero ¿somos capaces de racionalizar un problema tan complejo en el que intervienen tantos factores? Desde la economía conductual las respuestas a estas preguntas son escépticas y defienden, más bien, que los decisores tendemos a simplificar los problemas mediante una serie de principios desarrollados en el presente artículo, entre los que destacan el "isolation effect" y el "prominence effect" entre otros.

El objetivo del presente artículo es, por lo tanto, revisar aquellos principios que desde distintas áreas del marco teórico cuestionan nuestra capacidad de ser plenamente racionales para aplicarlos a un problema muy concreto: la compra de una vivienda. Así, entendiendo mejor las decisiones de los compradores, se entenderá mejor el funcionamiento del mercado inmobiliario en su conjunto. De esta forma, desde el punto de vista práctico, el presente artículo ayudará a todos los agentes (tanto compradores como vendedores) a optimizar sus decisiones.

Palabras clave: Comportamiento del Consumidor, Racionalidad Limitada, Marketing inmobiliario, Vivienda. 


\title{
Can we be rational buying a house? A Behavioral Economics approach
}

\begin{abstract}
Standard economic theory considers that consumer decisions tent to be rational and, that consumer success in rationality depends on the importance of the decision: the more transcendental is a decision, the more rational the consumer will be. Then, buying a house would require our best but, can we really be rational facing a so complex issue?

Behavioral economics is suspicious in this sense: decision makers tend to simplify problems using systematically some easy rules. This paper will describe how these rules, as the "isolation effect" and the "prominence effect", affect consumers during their housing purchase.

Therefore, the main objective is to look over to literature in order to show those effects that question our rationality during the housing purchase decision. Then, a better understanding of consumers decisions will lead a global understanding of real estate market. In that sense, this paper should give a piece of advice to buyers and sellers, in order to help them to optimize their decisions.
\end{abstract}

Key words: Consumer Behaviour, Bounded rationality, Real estate Marketing, Housing.

Sumario: 1. Introducción. 2. Antecedentes de la Economía Conductual. 3. El poder de la referencia. 4. ¿Qué valoramos y cómo? 5. Conclusiones.

\section{INTRODUCCIÓN}

La compra de una vivienda es una de las decisiones más importantes de nuestras vidas $\mathrm{y}$, por lo tanto, exige la mayor implicación posible por parte de los consumidores. Se espera entonces que estos compradores se comporten de manera especialmente racional, valorando todas las alternativas y sus consecuencias para escoger la mejor opción disponible en el mercado.

Este enfoque, compartido por las teorías económicas tradicionales, contrasta sin embargo con distintos puntos de vistas surgidos en los últimos años desde la perspectiva de la economía conductual. En efecto, frente a problemas complicados, nuestros esfuerzos por actuar como "homo oeconomicus" completamente racionales son muchas veces insuficientes. De hecho, en estos casos tendemos a servirnos de herramientas que nos permiten simplificar las decisiones y actuar, en lugar de quedarnos paralizados por la incertidumbre.

A partir de este planteamiento inicial, el objetivo de este artículo es el de ilustrar cómo funcionan algunos de estos principios que se emplean para simplificar y elegir cuando el problema es, como en caso de comprar una vivienda, inabarcable desde una perspectiva puramente racional. 
Para alcanzar estas metas, el artículo desarrollará tres apartados antes de aportar sus conclusiones. En primer lugar, se presentarán algunos antecedentes de la economía conductual. En segundo lugar, se profundizará en el poder que tienen las referencias previas a la hora de tomar una decisión de compra de una casa. Por último, se ilustrarán cuáles son los atributos determinantes a la hora de comprar una vivienda y cómo evaluarlos si pretendemos ser más racionales.

\section{ANTECEDENTES DE LA ECONOMÍA CONDUCTUAL}

\subsection{Los consumidores, ¿podemos en algún caso ser racionales?}

Las teorías económicas tradicionales insisten en considerar al consumidor como un "homo oeconomicus" perfectamente racional. Según estos principios de racionalidad global, antes de tomar una decisión valoramos todas las alternativas de compra, las clasificamos en una escala objetiva de valor o utilidad (donde además tenemos situados todo tipo de productos y alternativas) y escogemos finalmente la mejor opción.

Para comprar una casa, por ejemplo: consideraríamos todas las viviendas en venta, valoraríamos todos los aspectos de todas esas opciones (la localización, la luz, las inmediaciones, la distribución,...), compararíamos todos esos aspectos (entre sí, con su relevancia en el presupuesto, e incluso con su influencia en otro tipo de decisiones como el coche o las vacaciones que nos podríamos permitir) y, sólo después, escogeríamos la mejor alternativa del mercado.

Si alguna vez hemos comprado una casa, es fácil reconocer en nuestro comportamiento cierta voluntad por decidir de acuerdo a estas leyes de la racionalidad tradicional. Fue Herbert E. Krugman quién, en 1965, empleó por primera vez el término "involvement" a la psicología del consumidor. A partir de ahí, numerosos investigadores han trabajado sobre este concepto y han intentado medirlo, como Zaichkowsky (1985) al desarrollar la escala PII ("Personal Inveolvement Invetory") (Tabla 1) que permite (mediante 20 items evaluables en una escala Likert de 7 puntos) clasificar la implicación de un consumidor en una determinada decisión.

Tabla 1. Escala PII (Personal Involvement Inventory).

Traducción al castellano. Fuente: Beerli y Martín, 1999.

ESCALA DE ZAICHKOWSKY, "Personal Involvement Inventory”

Versión en ingles: $\quad$ Traducción al castellano:

1. Important / unimportant 1. Muy importante / poco importante 
2. Of no concern / Of concern to me

3. Irrelevant / Relevant

4. Means a lot to me / Means nothing to me

5. Useless / Useful

6. Valuable / Worthless

7. Trivial / Fundamental

8. Beneficial / Not beneficial beneficioso

9. Matters to me / Doestn't matter

10. Uninterested / Interested

11. Significant / Insignificant

12. Vital / Superfluous

13. Boring / Interesting

14. Unexciting / Exciting

15. Appealing / Unappealing

16. Mundane / Fascinating

17. Essential / Nonessential

18. Undesirable / Desirable

19. Wanted / Unwanted

20. Not needed / Needed
2. No me incumbe / sí me incumbe

3. Poco relevante / muy relevante

4. Significa mucho para mí / significa poco para mí

5. Poco útil / muy útil.

6. Muy valioso / poco valioso

7. Muy trivial / poco trivial

8. Muy beneficioso / poco beneficioso

9. Sí me conviene / no me conviene

10. Poco interesado en él / muy interesado en él

11. Muy significativo / poco significativo

12. Vital / innecesario

13. Poco interesante / muy interesante

14. Poco emocionante / muy emocionante

15. Muy sugerente / poco sugerente

16. Mundano / fascinante

17. Muy esencial / poco esencial

18. Muy atractivo / poco atractivo

19. Lo deseo mucho / no lo deseo

20. Muy necesario / poco necesario

A pesar de existir cierto consenso en el uso de esta escala PII para evaluar si un consumidor se implica más o menos en una decisión, no existe un criterio común a la hora de definir las consecuencias sobre su comportamiento. Así, el concepto de "involvement" ha servido como cajón de sastre para explicar distintos tipos de comportamiento, pero todos los puntos de vista coinciden en considerar que, cuanto mayor es nuestra implicación, mayor es nuestro esfuerzo por ser racionales. No es extraño por lo tanto que nos esforcemos por ser calculadores y objetivos frente a una decisión tan determinante como es la compra de una casa (no sólo por las implicaciones que tiene a nivel presupuestario sino también por la influencia que tiene sobre nuestro tipo de vida).

Si bien es fácil reconocer un esfuerzo por ser racionales en la compra de una casa, también es fácil reconocer en nuestra experiencia el fracaso en esa determinación. Alguno se sorprenderá argumentando que el baño de una opción es mucho más bonito que el de otra sin tener en cuenta que la diferencia en el precio permite una reforma que las iguale; los más concienzudos se verán haciendo una tabla Excel comparando factores cuyo equilibrio apenas tiene sentido ni siquiera para ellos mismos; y casi todos nos reconoceremos buscando argumentos racionales para justificar a nuestra pareja o a nosotros mismos por qué la vivienda que nos gusta es "objetivamente mejor" a las demás. 
Intentar ser objetivos, o justificar nuestras decisiones en base a una supuesta "racionalidad global" no deja de ser una coartada de cara a suavizar nuestra responsabilidad frente a posibles errores. Sin embargo, a poco que pensemos sobre ello, reconoceremos la incapacidad de resolver de manera racional la mayoría de los problemas complejos que nos rodean. Al comprar una casa por ejemplo, es muy difícil proyectar de manera objetiva la satisfacción que tendremos sobre el resultado de una reforma o, de manera mucho más evidente, es casi imposible aventurar la rentabilidad económica a veinte o treinta años de una decisión que tomamos en base a principios tan poco racionales como que "el ladrillo siempre es una buena inversión", "el precio de las casas nunca cae", o que "alquilar es tirar el dinero".

Herbert A. Simon (Premio Nobel en Economía en 1978) defendía que, frente a un problema complejo, bien sea porque no tenemos acceso a todos los datos, o bien porque no somos capaces de procesar una cantidad elevada de éstos al mismo tiempo, el caso es que es imposible valorar de manera racional todos los aspectos relevantes de todas las alternativas posibles. Según su Teoría de la Racionalidad Limitada de 1955, "a causa de las limitaciones psicológicas de los organismos (particularmente respecto a sus habilidades de computación y predicción), los esfuerzos del ser humano por ser racional pueden, como mucho, ser una aproximación vulgar y simplificada de la racionalidad global".

La idea de Simon (1955) no era desacreditar al consumidor tachándole de "limitado", todo lo contrario. La idea es aceptar el hecho de que estamos rodeados de problemas imposibles de resolver según los postulados económicos tradicionales, y empezar entonces a pensar cómo realmente actuamos en lugar de insistir en dictar cómo deberíamos actuar para que se cumplan las leyes de la economía tradicional. Posiblemente, las ideas de Simon han tardado en influir en los modelos econométricos y han quedado relegadas al ámbito de la "economía conductual" por la dificultad de predecir con ellas el comportamiento de todos los consumidores. Sin embargo, en todos estos años, muchos investigadores han puesto en relieve determinadas pautas que, lejos de esa racionalidad global del "homo oeconomicus", todos utilizamos de manera sistemática cuando nos enfrentarnos a problemas complicados.

Los principios de esa "economía conductual" cada vez están más extendidos en el ámbito de la investigación académica sin embargo, el ámbito de la empresa no sabe muchas veces en qué se traducen de forma concreta. Es por lo tanto objeto de este artículo ilustrar algunos de estos principios, explicando cómo afectan especialmente a la decisión de comprar una vivienda.

\section{2. "Cuentas mentales" utilizadas cuando valoramos nuestra propia casa}

Una particularidad fundamental de la vivienda como producto es su doble condición, por un lado se compra para su uso, por otro se pretende con ella hacer una buena inversión. Además, los compradores de una casa suelen ser al mismo 
tiempo vendedores de una anterior lo que, en principio, debería favorecer un comportamiento racional: los vendedores no tendrían por qué ser reticentes a vender en pérdidas ya que se beneficiarían como compradores de esa misma crisis del mercado; de la misma manera, en épocas de crecimiento, no tendrían por qué esperar mayores ganancias, ya que esas ganancias como vendedores se traducirían en pérdidas como inversores. A partir de este comportamiento, se podría esperar que la evolución del mercado inmobiliario fuese acorde con las leyes más clásicas, ajustándose precio, oferta y demanda de acuerdo con las condiciones económicas globales.

Para actuar de acuerdo a ese comportamiento racional, los consumidores deberían valorar todas las decisiones en una escala global de utilidad donde todas las alternativas pudiesen compararse con el objetivo de escoger la mejor. De ser puramente objetiva, esa escala no podría tratar de distinta manera las pérdidas y las ganancias, esto es que, en un mercado inmobiliario a la baja, las pérdidas como vendedores se compensarían en utilidad con las ganancias como compradores.

Este planteamiento no tiene sentido si tenemos en cuenta el principio de "aversión a las pérdidas" (Thaler, 1980) uno de los más aceptados desde la economía conductual. Según esta idea (anterior incluso a los postulados de Simon), los consumidores tendemos a ser conservadores afectándonos cualquier pérdida de manera desproporcionada respecto de las ganancias. No existiría entonces una escala global y objetiva de utilidad, sino un punto de partida a partir del cual codificaríamos las alternativas como positivas o negativas, como pérdidas o ganancias. Según Thaler (1985), este proceso de valoración de alternativas alejado del modelo económico tradicional se haría de acuerdo a una serie de "cuentas mentales" que explicarían en última instancia algunas características del mercado inmobiliario que contradicen el anterior enfoque económico:

- Segregación de ganancias (Gráfico 1): en relación a un punto de partida de referencia, cualquier ganancia, por pequeña que sea, es buena. Es más, ganar una pequeña cantidad es muy positivo si no pensábamos ganar nada, pero no lo es tanto si pretendíamos ganar mucho. Visto de otra forma, ganar diez veces 10 desde ese punto inicial de referencia es mucho mejor que ganar 100 de una sola vez: tenderemos entonces a "segregar las ganancias" para maximizar el valor que le damos a nuestras ganancias. Así, en un mercado inmobiliario creciente, los propietarios ven pronto que pueden amortizar su inversión y se apresuran a vender sin tener en cuenta que sus futuras casas también serán más caras. Las transacciones se multiplican rápidamente y los precios suben automáticamente explicando, de alguna manera, la creación de "burbujas" desde la perspectiva de la economía conductual. 


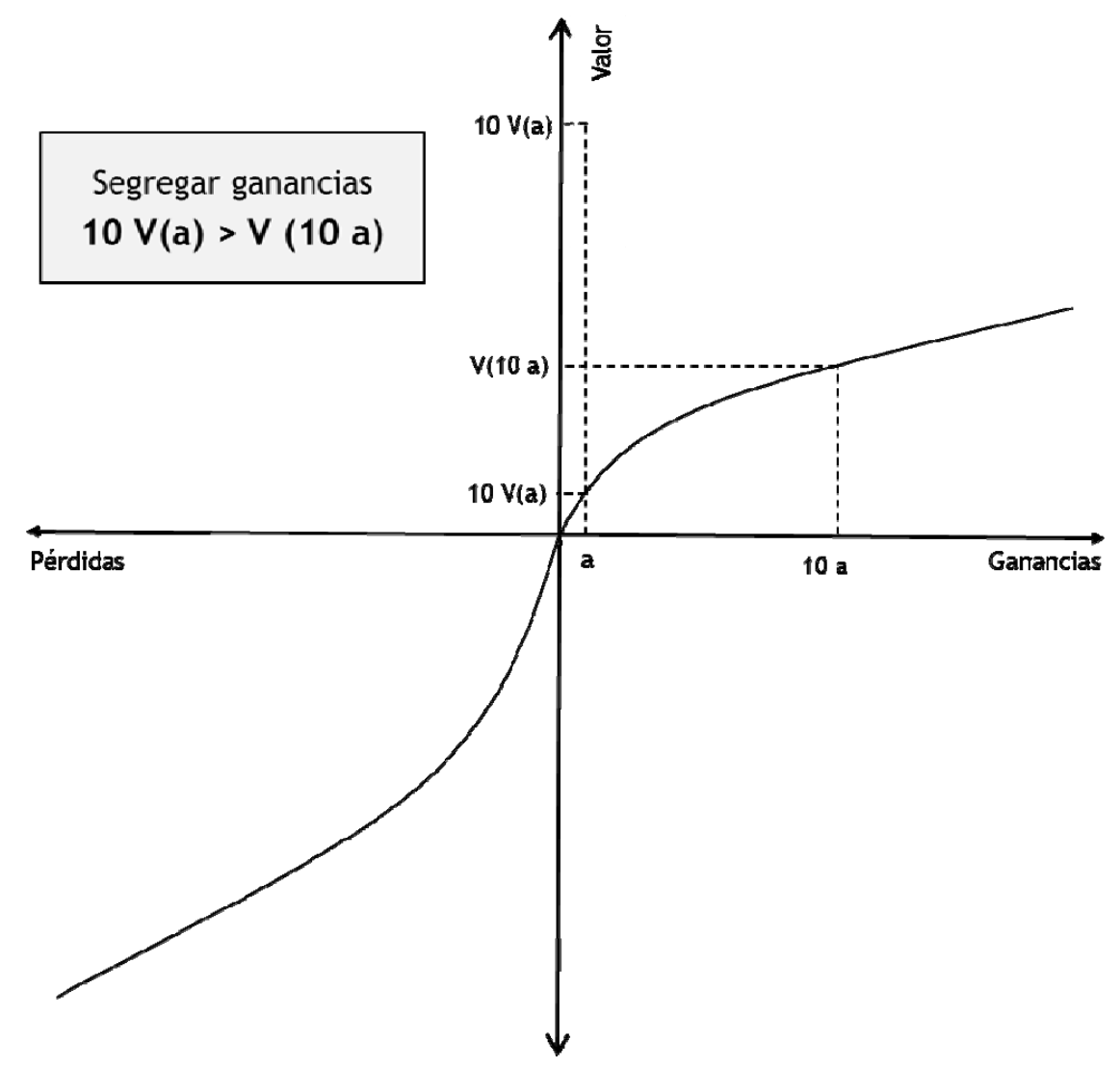

Gráfico 1. "Segregación de Ganancias", cuentas mentales de Thaler según la ecuación de valora Fuente: elaboración propia a partir de Thaler (1985)

- Integración de las pérdidas (Gráfico 2): de manera incluso más acentuada que para las ganancias, cualquier pérdida respecto a una referencia inicial es percibida por el consumidor como un auténtico drama. Así, frente a mercados a la baja, el propietario se resiste a vender, incluso cuando la venta irá asociada a una compra posterior donde sacar ventaja de la situación. No obstante, a partir de un momento, aguantar es insostenible. De hecho, resulta mucho más difícil asumir una derrota por la mínima que una goleada y, por lo tanto, los vendedores prefieren "integrar sus pérdidas", esto es bajar el precio de una vez por todas cuando el mercado ha caído ya en picado en lugar de hacerlo de manera progresiva. Así se explicaría, desde la perspectiva conductual, por qué es tan difícil "pinchar" una burbuja inmobiliaria y por qué, una vez se hace, se hace con caídas abruptas de los precios: en un primer momento de decadencia, los propietarios se resisten a 
perder, los compradores no encuentran ofertas acordes con la situación y no se deciden a comprar, las transacciones bajan hasta que ya es insostenible y hasta que los precios caen entonces de forma muy acusada.

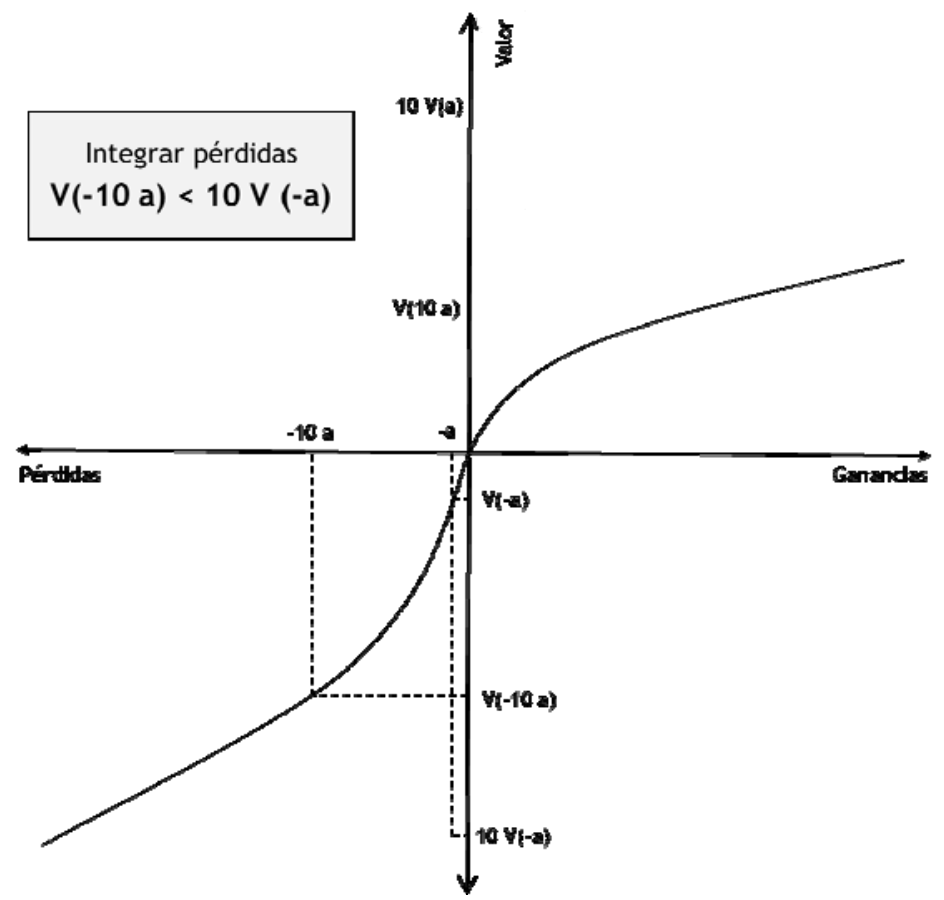

Gráfico 2. "Integración de Pérdidas", cuentas mentales de Thaler según la ecuación de valora Fuente: elaboración propia a partir de Thaler (1985)

- Cancelación de pérdidas y "Silver lining principle": Los principios de "segregación de ganancias" y de "integración de las pérdidas" enunciados por Thaler explican, desde la perspectiva del comportamiento del consumidor, por qué los propietarios tienden a ajustar los precios de manera más automática en mercados inmobiliarios al alza que lo que lo hacen en mercados a la baja. En última instancia, estos principios ofrecen una explicación sobre cómo se producen y se pinchan las burbujas inmobiliarias. Sin embargo, una vez conseguida una venta, los propietarios suelen comprar de nuevo una casa; es el momento de compensar pérdidas y ganancias. Los compradores, huyendo una vez más de las pérdidas, buscan como prioridad cancelarlas; es decir igualar lo perdido con lo ganado y dejar la cuenta a cero. No obstante, este cálculo no es siempre posible y, en ese caso, los consumidores solemos aplicar el "silver lining principle" que traducido al español de manera coloquial sería una especie de "sálvese quien pueda", es decir que si no somos capaces de pagar todas nuestras 
deudas, los consumidores preferimos mantener estas intactas y disfrutar de las ganancias por otro lado.

\section{EL PODER DE CUALQUIER REFERENCIA EN LA TOMA DE DECISIONES}

\subsection{Simplificaciones heurísiticas, decidir en la incertidumbre}

De acuerdo con la Teoría de la Racionalidad Limitada de Simon (1955), el también Premio Nobel de Economía Daniel Kahneman y su compañero de trabajo Amos Tversky han basado la mayor parte de su investigación en descubrir y explicar modelos de comportamiento que, lejos de los planteamientos econométricos clásicos, se repiten sistemáticamente en nuestra toma de decisiones. Así, en uno de sus más reconocidos trabajos, publicado en 1974, estos investigadores exponían una serie de "heurísticos", reglas sencillas que los consumidores emplean cada vez que sus decisiones son lo suficientemente complejas como para no poder abarcarse con la racionalidad global del "homo oeconomicus".

La idea de Kahneman y Tversky es sencilla. Cómo nuestras limitaciones cognitivas y la falta de información nos impiden ser racionales en la resolución de problemas complejos, utilizamos sistemáticamente reglas sencillas para simplificar esos problemas y decidir en la incertidumbre. Estas reglas, o "heurísticos" definidos por Tversky y Kahneman, son inicialmente las siguientes:

- El Heurístico de Representatividad: tendemos a calcular la probabilidad de que A pertenezca a $\mathrm{B}$, en función de cuánto A se parece a $\mathrm{B}$. De esta forma, tendemos a fiarnos de los estereotipos, y obviando en nuestros cálculos algunos datos importantes.

- Heurístico de Accesibilidad: tendemos a calcular la probabilidad de que ocurra un suceso en función de la facilidad de imaginárselo. De esta forma, los problemas más relevantes para la opinión pública suelen parecer muy comunes, mientras que subestimamos los problemas menos populares. Así, las muertes ligadas a accidentes de tráfico parecen mucho más frecuentes que las que están ligadas con problemas de diabetes cuando en realidad es todo lo contrario.

- Heurístico de anclaje y ajuste: cualquier valoración exige un punto de partida "anclaje" que se va "ajustando" durante la toma de decisión. Frente a problemas conocidos, los anclajes surgen de la experiencia previa y nos ayudan a mejorar en nuestras decisiones. No obstante, somos incapaces de valorar cualquier cosa sin estos puntos de partida $\mathrm{y}$, frente a nuevos 
problemas, escogemos cualquier referencia aunque no tenga nada que ver con el problema a tratar.

\section{2. ¿Cuánto pesan las referencias pasadas en la decisión de compra de una vivienda?}

Las "cuentas mentales" de Richard Thaler (1985) asumen la necesidad de un punto a partir del cual codificar las alternativas en términos de pérdidas y ganancias. La aplicación automática (y en la mayoría de los casos inconsciente) de esas reglas sencillas explicaría ciertas irregularidades del mercado inmobiliario que no se comporta siempre como debería hacerlo de acuerdo con las leyes económicas tradicionales. No obstante, a pesar de explicar cómo procesamos alrededor de un punto de origen, esas "cuentas mentales" no explican cómo fijamos y cómo adaptamos esas referencias iniciales.

Al igual que Thaler; Tversky y Kahneman (1979) entienden, según exponen en su Teoría Prospectiva, que son necesarias referencias en cualquier valoración que hacemos de un producto o de una alternativa. Los consumidores, al necesitar de ellas, buscan esas referencias o "anclas" nada más empezar el proceso de decisión. El éxito de la decisión no depende sólo de ese "anclaje" previo sacado de los conocimientos más cercanos del consumidor, sino también de un proceso posterior de "ajuste" que permite adaptar las expectativas durante el proceso de compra; dicho de otra manera, los errores pueden provenir tanto de un "anclaje" incoherente como de un "ajuste" insuficiente.

Desde el enunciado de Kahneman y Tversky, el uso de puntos de referencia en las valoraciones se ha estudiado desde la perspectiva de las finanzas, el mercado laboral, los seguros, y por supuesto en las decisiones de compra de viviendas. Sin duda, el "anclaje y ajuste" es, entre los heurísticos de Kahneman y Tversky, el que más se han investigado, y sus aplicaciones son directas en el mercado inmobiliario. Obviamente, como vendedores, los propietarios están "anclados" en el precio en el que compraron anteriormente sus casas y deben ajustarlo, con mayor o menor éxito, a las variaciones que desde entonces ha sufrido el mercado. La vivienda de origen también es una poderosa ancla para los compradores, que comparan siempre sus nuevas casas con sus casas anteriores. Según la perspectiva de Tversky y Kahneman (1974), esa comparación es natural y no es peligrosa siempre y cuando el anclaje posterior que nos ayuda a tomar la decisión final se haga correctamente. No obstante, este ajuste no es siempre evidente.

En una investigación académica llevada a cabo con datos reales de inquilinos de Estados Unidos, se ponía efectivamente en relieve que, a la hora de mudarse de casa, los inquilinos buscaban sistemáticamente las ofertas con un rango de precio basado en su anterior vivienda (Simonsohn y Loewenstein, 2006). Lo curioso de la investigación era comprobar las dificultades que estos inquilinos tenían a la hora de ajustar ese rango de precio a cambios en el mercado. Así, cuando una mudanza se produce entre dos ciudades distintas resulta extremadamente difícil adaptar el 
presupuesto a las nuevas condiciones: al pasar de una ciudad más barata a una más cara, los inquilinos tienden a buscar pisos de precio más bajo independientemente de haber experimentado subidas salariales; del mismo modo al mudarse de una ciudad más cara a una más barata se buscan pisos más caros de lo que nuestra situación económica en la nueva ciudad recomendaría.

El mercado inmobiliario tiene características muy concretas. Por ejemplo, al mismo tiempo que se comprar una casa, se suele vender una vivienda anterior. Los compradores son a la vez vendedores; las ventajas de comprar a la baja son al mismo tiempo inconveniente en la venta y, por lo tanto, cabría esperar que la adaptación de sus expectativas al mercado fuese inmediata (como sugieren las teorías económicas estándar). Sin embargo, a la hora de vender sus casas, los propietarios suelen estar anclados fuertemente al precio original de compra (Bucchianeri \& Minson, 2013), y suelen estar más influenciados por sus expectativas de cuando compraron la casa que a las fluctuaciones reales del mercado. (Benítez-Silva et al., 2008).

De esta forma se da respuesta, desde la perspectiva del comportamiento del consumidor, a dinámicas globales del mercado inmobiliario. Mientras que los precios suben, los vendedores adaptan enseguida sus expectativas $\mathrm{y}$, queriendo mayores ganancias, suben y suben los precios rápidamente, alimentando la aparición de burbujas. Cuando los precios bajan, los vendedores se resisten y evitan las pérdidas hasta que éstas son ya muy grandes: las burbujas se rompen de golpe (Benítez-Silva et al., 2008; Genesove \& Mayer, 2001).

El anclaje y ajuste ha servido a menudo para explicar aspectos relacionados con variables cuantitativas como el precio. Pero también existen ejemplos que muestran cómo aspectos subjetivos como la valoración de una experiencia están tan al anclaje cómo las valoraciones alrededor del precio de un producto (Ariely et al., 2006). De hecho, los consumidores pueden en determinados productos y marcas ser mucho más sensibles a variaciones respecto a aspectos cualitativos que a aspectos cuantitativos (Hardie et al., 1993). No hay que olvidar que la dependencia a un punto de referencia inicial es un mecanismo psicológico y por lo tanto puede estar influenciada por una suerte de afecto inicial que condiciona los juicios posteriores (King \& Slovic, 2014; Slovic et al., 2007). No es por lo tanto extraño distinguir entre un aspecto más mecánico y otro más psicológico a la hora de estudiar la influencia que las referencias previas tienen sobre nuestros juicios (Trautmann \& Kuilen, 2015).

Sin embargo, y a pesar de reconocerse que la influencia de los puntos de referencia iniciales puedes ser más fuerte para valoraciones cualitativas (Hardie et al., 1993) especialmente en el caso de las decisiones de compra de una vivienda (Dunn et al., 2003; Paraschiv \& Chenavaz, 2011), apenas hay investigaciones que profundicen en este aspecto del heurístico originalmente enunciado por Tversky \& Kahneman (1974). 
La necesidad de referencias a la hora de valorar una decisión es tan grande que el poder de algunas anclas es inmune a cualquier proceso de ajuste. Esta observación es particularmente cierta ( $\mathrm{y}$ ha estado particularmente estudiada) alrededor de nuestro comportamiento frente a los precios. De hecho, algunos investigadores han contrastado como, frente a nuevos productos, las referencias que adoptamos pueden ser arbitrarias e incluso absurdas; pero a partir de ahí lo curioso es comprobar cómo nos esforzamos por adaptarlas inútilmente para obtener respuestas objetivas. Este principio, llamado de "arbitrariedad coherente" (Ariely et al., 2003; ) afecta sin duda a las valoraciones que hacemos de las viviendas, pero no explica qué atributos de éstas son los que nos importan a la hora de tomar una decisión de compra.

\section{4. ¿QUÉ VALORAMOS Y CÓMO HACERLO PARA SER MÁS "RACIONALES"?}

\section{1. ¿Qué atributos de una casa valoramos cuando valoramos una casa?}

El heurísticos de "anclaje y ajsute" (Tversky y Kahaneman, 1974) explica hasta qué punto son necesarias referencias cuando enfrentamos cualquier valoración. La aplicación de este heurístico es directa para explicar dinámicas de precios $\mathrm{y}$, de hecho, se ha utilizado para explicar determinados comportamientos en este sentido de vendedores y compradores en el mercado inmobiliario (Benítez-Silva et al., 2008; Bucchianeri \& Minson, 2013; Genesove \& Mayer, 2001; Simonsohn y Loewenstein, 2006). No obstante, la aplicación del heurístico definido originalmente por Tversky y Kahneman también explica el comportamiento de los a la hora de valorar otro tipo de atributos cualitativos, ligados a con la experiencia de los consumidores (Ariely et al., 2006; Hardie et al., 1993; King \& Slovic, 2014; Slovic et al., 2007). Así, en la medida en que reconocemos que la elección de una vivienda está estrechamente ligada a la valoración que se hace de sus aspectos más cualitativos, conviene detenerse y clarificar cuáles son éstos: revisando la literatura y definiendo qué atributos valoramos cuando elegimos una casa.

La primera vez que el New York Times utiliza la expresión "consumidor de viviendas" es en 1941. Antes, los compradores de casas eran clientes particulares que se relacionaban de manera personal con constructores y contratistas; a partir de entonces, los promotores debieron construir casas sin conocer previamente a sus compradores es decir que debieron plantearse de antemano cómo debía ser el producto para satisfacer las demandas de sus clientes. De hecho, el problema principal es que la vivienda es un producto especialmente indivisible: tiene muchas características que, aunque satisfacen distintas exigencias de los compradores, no pueden modificarse independientemente. Dicho de otra manera, a los ojos del comprador, una casa puede destacar por sus preciosas vistas y otra opción por un 
estupendo jardín, pero no podemos ofrecerle comprar las vistas de la primera de las opciones para ponerlas en el jardín de la segunda.

Si bien crítico en el caso de la vi vivienda, la indivisibilidad de los productos es un problema bastante extendido en economía. La Teoría de los Precios Hedónicos, enunciada por Rosen (1974) es una solución bastante ingeniosa a este problema y ha sido utilizada a menudo para explicar el mercado inmobiliario. La idea es sencilla, por muy indivisible que sea un producto podemos identificar algunas de las características que lo componen y pensar que el precio total del mismo es una función matemática que depende de todas esas características. A partir de ahí, y mediante datos estadísticos, una simple regresión matemática permite estimar cuánto cada atributo ha influido en la función global o, dicho de otra manera, qué peso tiene cada característica considerada en el precio final. Volviendo al ejemplo anterior, no podemos comprar las vistas y cambiarlas de casa, pero sí podemos valorar cuánto esas vistas influyen en el precio de la casa en la que se encuentran.

No es de extrañar el éxito de la Teoría de los Precios Hedónicos en el estudio académico del mercado inmobiliario (Fletcher et al., 1995). Una vivienda se valora, por ejemplo, por su ubicación, su superficie, sus espacios exteriores... todas esas características son inseparables del producto final, pero esta Teoría permite conocer cuánto influye cada una de ellas en el valor total. No obstante, a pesar de su aparente utilidad, la aplicación del método enunciado por Rosen encuentra algunas limitaciones al aplicarse al mercado inmobiliario (Goodman, 1978). En primer lugar, las regresiones matemáticas permiten asegurar hasta qué punto las características consideradas afectan al precio final, pero no aseguran que no influya alguna otra característica no considerada en el modelo. En segundo lugar, y quizá de manera más importante, para sacar una conclusión definitiva acerca de qué influye y de en qué medida lo hace sobre nuestras decisiones de compra, el método exige un equilibrio en la función que determina el precio de la vivienda. Dicho de otra manera, podemos hacer una foto fija de qué factores afectan y de qué manera lo hacen sobre el precio de una vivienda, pero si esos factores cambian o si el poder de éstos varía, nuestros esfuerzos son inútiles.

Esta última limitación de la Teoría de los Precios Hedónicos impide en última instancia encontrar una fórmula que defina los gustos y preferencias de todos los consumidores a la hora de comprar una vivienda, pero al mismo tiempo permite contrastar las diferencias de estos consumidores dependiendo del contexto (del momento y la localización). Si bien a nivel internacional son muchas las investigaciones académicas basadas en la Teoría de los Precios Hedónicos para conocer mejor las preferencias de los compradores en determinados "sub-mercados" estables, en este método no está muy extendido todavía en España. No obstante, las aplicaciones más directas de esta Teoría de los Precios Hedónicos llevadas a cabo por investigadores españoles (Núñez et al., 2007 y Gallego, 2004) coinciden con las investigaciones internacionales y con los consejos dados a los compradores por los 
medios de divulgación (como idealista.com) en distinguir ciertos aspectos que afectan indiscutiblemente a la valoración de una vivienda (Tabla 2):

- Ubicación: la situación global dentro de la ciudad (barrio) como la localización específica (proximidad a comercios, servicios y transportes públicos...)

- Exteriores: tanto los espacios exteriores a los que vuelca la vivienda como los servicios exteriores a esta como las zonas comunes, garajes, etc.

- Calidad material: la situación constructiva de la casa, los materiales, las reformas necesarias, etc.

- Calidad espacial: la distribución, la luminosidad, etc.

Tabla 2. Atributos importantes en la valoración de una casa, comparación según la fuente.

$$
\text { ATRIBUTOS A VALORAR EN UNA VIVIENDA PARA SU COMPRA }
$$

\section{“Aproximación a la} valoración inmobiliaria mediante la metodología de los precios hedónicos" (Núñez, Ceular y Millán, 2007)

\section{1. Índice Arquitectónico} externo, según la disponibilidad de determinados servicios (piscina, pista de tenis, jardines,...)

2. Índice Arquitectónico interno, definido en función de la antigüedad del edificio, la disponibilidad de ascensor o la existencia de terraza. 3. Índice de Anejos, si tiene o no tiene garaje y trastero. 4. Índice de Ubicación, definido en base al barrio donde se ubica el edificio y el nivel de renta de la zona.

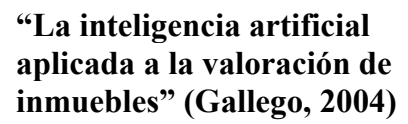

"La inteligencia artificial aplicada a la valoración de inmuebles" (Gallego, 2004)

10 cualidades que convierten una casa en una buena casa (idealista.com, 2012)

\section{Distancia al centro de la ciudad.}

2. Vía: vía de acceso al centro de la ciudad.

3. Tamaño: tamaño del municipio.

4. Categoría: categoría de la construcción.

5. Antigüedad: antigüedad de la construcción.

6. Conservación: estado de conservación de la construcción.

7. Superficie construida.

8. Terraza: superficie de la terraza.

9. Entorno: situación en el municipio, distrito o barrio.

10. Interior: vivienda interior

11. Planta: número de planta

12. Anejos: jardines, piscina,
1. Localización, localización, localización: "las famosas tres "l" del mercado inmobiliario.

2. Cercanía de colegios.

3. la posición de la vivienda en el edificio o urbanización. 4. Seguridad del vecindario.

5. Servicios accesibles a pie.

6. Los vecinos.

7. No ser la mejor casa del edificio, "porque estarás rodeado de viviendas peores y puede ahuyentar a futuros compradores"

8. Necesidad de reparaciones

9. Mantendrá su valor. 10. Impuestos y tasas.

$\cdots$

Fuente: elaboración propia a partir de las fuentes citadas. 


\section{2. ¿Elegir o comparar, cómo ser más racional?}

La Teoría de los Precios Hedónicos permite, en contextos concretos, determinar hasta qué punto distintas características de la vivienda influye en los precios del mercado inmobiliario. Esta herramienta es por lo tanto muy útil a la hora de generalizar, pero sigue sin explicar cómo realmente los consumidores valoran los distintos atributos de una casa durante el proceso de búsqueda y, sobre todo, no explica en qué medida estos atributos condicionan la decisión final de compra (muchos de ellos cualitativos). Dicho de otra manera, de la aplicación de la Teoría de los Precios Hedónicos se puede desprender la importancia que los espacios exteriores, las calidades materiales o la calidad espacial tienen en el precio en un determinado barrio de una determinada ciudad, pero ¿hasta qué punto estas características han condicionado la compra de una casa en concreto?

En este sentido, las recientes investigaciones en el ámbito de la economía conductual coinciden en que el fenómeno de anclaje y ajuste, definido inicialmente por Tversky y Kahneman (1974), afecta no sólo a las características cuantitativas de los productos (como el precio) sino también a las características cualitativas de los mismos. Así, la luz, las calidades de los materiales, o el entorno y las zonas comunes de una vivienda no se valoran en términos globales y objetivos, sino que necesitan compararse con algún punto previo de referencia. De esta forma, la vivienda de origen no sería sólo un poderoso "ancla" a la hora de decidir el precio a pagar en una futura casa, sino que condicionaría también la valoración que se hace de todos esos otros aspectos cualitativos de las opciones consideradas.

A partir de un anclaje inicial, antes de tomar cualquier decisión, se produce un ajuste. En el caso de la vivienda, el proceso de búsqueda es largo y conlleva la visita de varias alternativas antes de decidirse por una. Estas visitas parecen una oportunidad idónea a la hora de llevar a cabo un ajuste coherente con las necesidades; no obstante, las investigaciones académicas recientes sugieren que no tiene por qué ser el caso: si bien somos capaces de anclar y ajustar varias características de un producto, solemos ser más sensibles a las variaciones de alguna de ellas (Hardie et al., 1993). Dicho de otra forma, si nuestra vivienda de anclaje, o la primera que visitemos en nuestra búsqueda, destaca mucho en un determinado factor, este factor será mucho más determinante en el resto del proceso. Si, por ejemplo, la primera vivienda que consideremos tiene mucha luz, las demás viviendas parecerán oscuras y las diferencias en otros factores cómo las calidades pueden pasar desapercibidas.

Este fenómeno, de hecho, es coherente con el efecto aislamento, "isolation effect", también definido por Kahneman y Tversky (1979): "generalmente la gente descarta los componentes compartidos por las distintas alternativas consideradas. Esta tendencia, conocida como "isolation effect" conduce a establecer preferencias inconsistentes, que dependen de la manera en que son presentadas las decisiones". Según este efecto, los consumidores tendemos a elegir en función de las características que más distingan a las distintas opciones de compra, obviando 
pequeñas diferencias en otros factores que, a la larga, pueden ser mucho más determinantes.

El efecto aislamiento puede ser especialmente crítico en un proceso de compra como el de una casa donde la decisión se basa, muchas veces, en elegir entre un grupo de alternativas bastante dispares. En efecto, al comprar una casa, se suele, en primer lugar, filtrar las opciones con respecto a un rango de precio, una localización y una superficie determinada. A partir de ahí se visitan algunas alternativas y se decide eligiendo entre éstas según un procedimiento que invita a valorar aquellas características que más las distinguen (obviando, como expone el "isolation effect", pequeñas diferencias en determinados factores que, en realidad, podrían ser mucho más importantes).

No obstante, frente al efecto aislamiento, el propio Tversky, esta vez junto con Sattah y Slovic (1988), contrastaron otro fenómeno que llamaron "prominence effect". Este efecto prominencia distingue entre dos tipos de decisiones: las que consisten en elegir entre varias alternativas (donde premiaría el efecto aislamiento), y las decisiones que exigen comparar entre sí los distintos atributos que definen a las alternativas (donde el peso del factor prominente no sería tan determinante). De esta forma por ejemplo, si tenemos que elegir entre tres viviendas cuyas diferencias en luz son muy importantes mientras que sus diferencias en los espacios comunes son mínimas, tenderemos a escoger aquella más luminosa. No obstante, si transformamos el problema en una comparación de atributos, es decir, si nos preguntásemos qué tipo de equipamientos comunes compensarían la falta de luz de una de las opciones, automáticamente la luz dejaría de ser un factor tan prominente en nuestra decisión.

De hecho, algunos investigadores que han trabajado sobre este efecto prominencia coinciden en señalar que, una vez comparamos las distintas características de un producto unas con otras, los consumidores somos capaces de establecer "puentes de proporcionalidad" entre esos atributos (Carmon y Simonson, 1998); esto es que relacionamos cuánto de un factor nos merece la pena otro. Dicho de otra manera y a modo de consejo para compradores de casas, si en algún momento comparando opciones nos sorprendemos dándole mucha importancia a un determinado factor (como puede ser la luz) basta con compararlo con el resto de atributos (como las zonas comunes, la calidad de los materiales o la distribución) para, automáticamente, ser menos impulsivos en nuestra decisión.

\section{CONCLUSIONES}

Elegir una vivienda es un problema crucial en nuestras vidas y, por lo tanto, debemos dedicarnos a resolverlo con el mayor compromiso. Esta implicación, según las teorías tradicionales, se traduciría en un comportamiento puramente 
racional donde, buscaríamos y procesaríamos toda la información necesaria para poder ordenar todas las opciones del mercado hasta escoger la mejor de ellas.

¿Somos capaces de actuar de acuerdo a esta racionalidad global de los modelos econométricos tradicionales? Desde la economía conductual parece claro que no: ni toda la información que afecta al éxito o el fracaso de nuestra decisión suele estar disponible, ni de estarlo seríamos capaces de procesarla (Simon, 1955). Por lo tanto, frente a problemas tan complicados como elegir una casa, necesitamos herramientas de simplificación si no queremos quedarnos paralizados.

La primera simplificación es clara. En lugar de valorar todas las alternativas en un momento para escoger la mejor después, lo que hacemos es fijar un punto inicial de referencia que vamos adaptando según nos enfrentamos a la realidad del mercado. Este fenómeno, de "anclaje y ajuste" (Tversky y Kahneman, 1974) es básico para entender la importancia de planificar las visitas cuando estamos buscando casa: si las primeras opciones son malas, las siguientes parecerán artificialmente malas y viceversa. Además, este principio es determinante en la medida en que lo aplicamos a la valoración de todo tipo de atributos, a los cuantitativos como puede ser el precio, pero también a los cualitativos como lo es lo luminosa que es una casa.

De hecho, la vivienda está definida por muchos factores de muy distinto tipo que deberían influir en cualquier decisión de compra. No obstante, no está claro que todos esos atributos afecten de manera proporcionada; algunos investigadores sugieren que sólo nos fijamos en los atributos más significativos, aquellos que más distinguen a las opciones. Según este "efecto aislamiento" (Kahneman y Tversky, 1979), si por ejemplo una vivienda destacase de un grupo por su luz natural, este factor se convertiría inmediatamente en el factor determinante de la decisión de compra. Para evitar menospreciar la importancia de otros factores la economía conductual también tiene su receta, según el "efecto prominencia" (Tversky et al, 1988), basta plantearse cuánto de ese factor prominente renunciaríamos para mejorar el resto de atributos, y automáticamente seremos más proporcionales en nuestra valoración.

\section{BIBLIOGRAFÍA}

ARIELY, D., LOEWENSTEIN, G., \& PRELEC, D. (2006). "Tom sawyer and the construction of value". Journal of Economic Behavior \& Organization, 60(1), p. 1-10.

BENÍTEZ-SILVA, H., EREN, S., HEILAND, F., \& JIMÉNEZ-MARTÍN, S. (2015). "How well do individuals predict the selling prices of their homes?" Journal of Housing Economics, 29, p.12-25 
BUCCHIANERI, G. W., \& MINSON, J. A. (2013). “A homeowner's dilemma: Anchoring in residential real estate transactions". Journal of Economic Behavior \& Organization, 89, p. 76-92.

CARMON, Z., \& SIMONSON, I. (1998). "Price-quality trade-offs in choice versus matching: New insights into the prominence effect". Journal of Consumer Psychology, 7(4), p. 323-343.

DUNN, E. W., WILSON, T. D., \& GILBERT, D. T. (2003). "Location, location, location: The misprediction of satisfaction in housing lotteries". Personality and Social Psychology Bulletin, 29(11), p. 1421-1432.

FLETCHER, M., MANGAN, J., \& RAEBURN, E. (2004). "Comparing hedonic models for estimating and forecasting house prices". Property Management, 22(3), p. 189-200.

GALLEGO MORA-ESPERANZA, J. (2004). "La inteligencia artificial aplicada a la valoración de inmuebles, un ejemplo para valorar Madrid". CT: Catastro, 50, p. 51-68.

GENESOVE, D., \& MAYER, C. (2001). "Loss aversion and seller behavior: Evidence from the housing market". The Quarterly Journal of Economics, 116(4), p. 1233-1260.

GOODMAN, A. C. (1978). "Hedonic prices, price indices and housing markets". Journal of Urban Economics, 5(4), p. 471-484.

HARDIE, B. G. S., JOHNSON, E. J., \& FADER, P. S. (1993). "Modeling loss aversion and reference dependence effects on brand choice". Marketing Science, 12(4), p. 378-394.

KAHNEMAN, D., \& TVERSKY, A. (1979). "Prospect theory: An analysis of decision under risk". Econometrica: Journal of the Econometric Society, 47, p. 263-291.

KING, J., \& SLOVIC, P. (2014). "The affect heuristic in early judgments of product innovations". Journal of Consumer Behaviour, 13(6), p. 411-428.

KRUGMAN, H. E. (1965). "The impact of television advertising: Learning without involvement”. Public Opinion Quarterly, 29(3), p. 349-356.

NÚÑEZ TABALES, J., CEULAR VILLAMANDOS, N., \& MILLÁN VÁZQUEZ DE LA TORRE, M.G. (2007). "Aproximación a la valoración inmobiliaria mediante la metodología de precios hedónicos (MPH)". Conocimiento, Innovación y Emprendedores: Camino Al Futuro, p. 2688-2701.

PARASCHIV, C., \& CHENAVAZ, R. (2011). "Sellers' and buyers' reference point dynamics in the housing market". Housing Studies, 26(03), p. 329-352.

ROSEN, S. (1974). "Hedonic prices and implicit markets: Product differentiation in pure competition". Journal of Political Economy, 82(1), p. 34-55.

SIMON, H. A. (1955). "A behavioral model of rational choice". The Quarterly Journal of Economics, 69(1), p. 99-118. 
SIMONSOHN, U., \& LOEWENSTEIN, G. (2006). "Mistake\# 37: The effect of previously encountered prices on current housing demand*". The Economic Journal, 116(508), p. 175-199.

SLOVIC, P., FINUCANE, M. L., PETERS, E., \& MACGREGOR, D. G. (2007). "The affect heuristic". European Journal of Operational Research, 177(3), p. $1333-1352$.

THALER, R. (1980). "Toward a positive theory of consumer choice. Journal of Economic Behavior \& Organization", 1(1), p. 39-60.

THALER, R. (1985). "Mental accounting and consumer choice. Marketing Science, 4(3)", p. 199-214.

TRAUTMANN, S. T., \& KUILEN, G. (2015). "Reserve price and competing bids: Reference points for product evaluations in online auctions?" Journal of Consumer Behaviour, 14(4), p. 285-296.

TVERSKY, A., \& KAHNEMAN, D. (1974). "Judgment under uncertainty: Heuristics and biases." Science, 185, p. 1124-1131.

TVERSKY, A., SATTATH, S., \& SLOVIC, P. (1988). "Contingent weighting in judgment and choice". Psychological Review, 95(3), p. 371-384.

ZAICHKOWSKY, J. L. (1986). "Conceptualizing involvement". Journal of Advertising, 15(2), p. 4-34. 